Post-print of (paper) Biophys. Chem., 2006, 122, 27-35.

Dynamics of bolaamphiphilic fluorescent polyenes in lipid bilayers from polarization emission spectroscopy

A. Ulises Acuña, Francisco Amat-Guerri, Ernesto Quesada, Marisela Vélez.

Received 12 December 2005; received in revised form 9 February 2006; accepted 10 February 2006

DOI: $10.1016 /$ j.bpc.2006.02.004

\title{
Dynamics of bolaamphiphilic fluorescent polyenes in lipid bilayers from polarization emission spectroscopy
}

\author{
A. Ulises Acuña a, $\square$, Francisco Amat-Guerri b, Ernesto Quesada a, Marisela Vélez a \\ a Instituto de Química-Física “Rocasolano”(CSIC), Serrano 119, 28006-Madrid, Spain \\ b Instituto de Química Orgánica (CSIC), Juan de la Cierva 3, 28006-Madrid, Spain
}

\begin{abstract}
The rotational motions of the biamphiphilic polyenes (bolapolyenes) dimethyl all-(E)-octacosa10,12,14,16,18-pentaenedioate (DE28:5) and dimethyl all-(E)-tetratriaconta-13,15,17,19,21pentaenedioate (DE34:5), with head-to-head distances of 34 and $42 \AA$, respectively, have been examined by fluorescence anisotropy methods. The membrane-spanning bolapolyenes, which contain a central emitting pentaene group tethered to two methoxycarbonyl opposite polar heads by symmetric C8 (DE28:5) and C11 (DE34:5) polymethylene chains, were dispersed in lipid bilayers of DPPC or DMPC, and the stationary and picosecond-resolved emission was recorded as a function of temperature. In fluidphase DMPC bilayers, three relaxation times could be determined, assigned to fast ( 0.2 and $2 \mathrm{~ns})$ singlebond isomerization processes localized on the alkyl chains, and to whole-molecule oscillations $(\sim 11 \mathrm{~ns})$, respectively. The anisotropy decay parameters were further analyzed in terms of a diffusive model for wobbling in a Gaussian ordering potential, to assess the anchoring effect of the symmetric polar heads. In this way, the average rotational diffusion constant of the bolapolyenes, $\mathrm{D} \perp$, could be estimated as $0.022-$ $0.026 \mathrm{rad} 2 \mathrm{~ns}-1$ (DMPC bilayers, $35^{\circ} \mathrm{C}$ ), a value that is only $1 / 3$ of that corresponding to the related pentaene fatty acid spanning a single membrane monolayer. In contrast, the amplitude of the equilibrium orientational distribution ( $\theta$ half-cone $50^{\circ}$ ) is very similar for both the transmembrane and the singleheaded polyenes. The reorientational oscillations of the central emitting group in the bolapolyenes necessarily would produce large-amplitude $(2-5 \AA)$ and very fast (ns) translational motions of the polar heads.
\end{abstract}




\section{Introduction}

The structure and dynamics of artificial lipid bilayers made up from ordinary, single-headed phospholipid molecules have been characterized in great detail using a variety of experimental and theoretical methods [1-5]. Apart from their intrinsic interest, these model membranes have been successfully used to reproduce many of the physical and chemical phenomena taking place in the complex and specialized lipid matrix of natural membranes. The efficiency and characteristic time domain of the biological processes often require the lipid membrane to be in a fluid, liquid-like state. Fluidity, in this context, results from thermal fluctuations in the position (lateral and transverse) and angular orientation of individual amphiphilic lipid molecules. Lipid reorientational motions, in particular, are a complex superposition of head group oscillation, fast conformational changes of the acyl chains and wholemolecule rotation. Since these processes may take place in different time ranges, the experimental recording of the motions of bilayer lipid molecules has been carried out by a number of complementary techniques [3-5]. As a result of that, it is now possible to understand the main dynamic properties of the superstructure in terms of those of its individual components.

On the other hand, the lipid membranes of Archaebacteria, a rather unusual type of organisms, are often

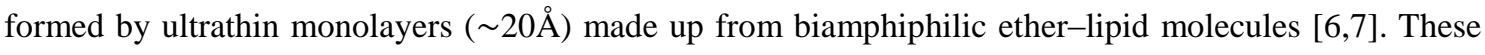
lipids (also know as bolalipids) consist of a hydrophobic skeleton joining two hydrophilic distal head groups. This specialized structure provides the bacteria with additional stability against harsh environments, while preserving the functional properties of the membrane. In addition, synthetic bolaamphiphilic molecules are currently being produced and studied in great detail, with a large variety of structures and functional groups [8]. However, specific information on the dynamic properties of both the natural and synthetic bipolar lipids is still quite limited (see Ref. [9] for a recent comprehensive review of bolalipid and monolayer dynamic properties). The lateral mobility of a fluorescent derivative of a natural tetraether bolalipid embedded in lipid bilayers has been determined by fluorescence recovering after photobleaching techniques [10]. The translational diffusion coefficient of this biamphiphilic molecule was not very different $(\sim 70 \%)$ from that of a regular single-headed phospholipid. Similar studies concerning biamphiphile rotational motions have not been carried out, as far as we are aware.

The methods of time-resolved fluorescence depolarization, which are frequently used to characterize the angular motions of lipid probes in artificial bilayers and cell membranes [11-14], may also be applied to the study of bolaamphiphile rotational dynamics. Briefly, the method is based on recording the ns decay of the fluorescence anisotropy, $\mathrm{r}(\mathrm{t})$, of a lipophilic probe molecule embedded in the bilayer. In the simplest case, it is observed that the anisotropy decays down to a time-independent value, ro (in the ns range). The kinetic parameters of the decay (anisotropy relaxation times, $\phi i)$ depend on the rate of angular motions [13-17] of the probe (rotational diffusion constant, Dr), while the ro value is related to the ordering effect of the bilayer structure on the emitting group [18-20]. The interpretation of this kind of experiments is simpler for rigid, rod-like fluorophores with electronic transition moments parallel to the long molecular axis and with a well-defined transverse location in the bilayer. Fluorescent polyenes that closely approach these properties are charged analogs of 1,6-diphenyl-1,3,5-hexatriene [21] and the linear 
single-headed polyenes parinaric [22] and t-COPA [23,24] fatty acids, with four and five conjugated double bonds, respectively. With the purpose of determining the dynamic properties of bolaamphiphilic lipids based on these methods, we synthesized [25] several emitting bolalipid analogs, with a central polyene system linked to $\alpha, \omega$ polar headgroups by symmetric polymethylene connectors (Scheme 1). Short polyenes, with four or five conjugated double bonds, are weak emitters and may photodegrade under intense irradiation. However, these groups introduce a minimum perturbation in the lipid system [23,24], compared with standard lipophilic polyaromatic tags. For the present study we selected two bipolar $\alpha, \omega$-di(methoxycarbonyl) analogs of the pentaene fatty acid t-COPA, code-named DE28:5 and DE34:5 (the numbers before and after the colon indicate, respectively, the total number of carbon atoms and the conjugated double bonds of the molecule, see Scheme 1).
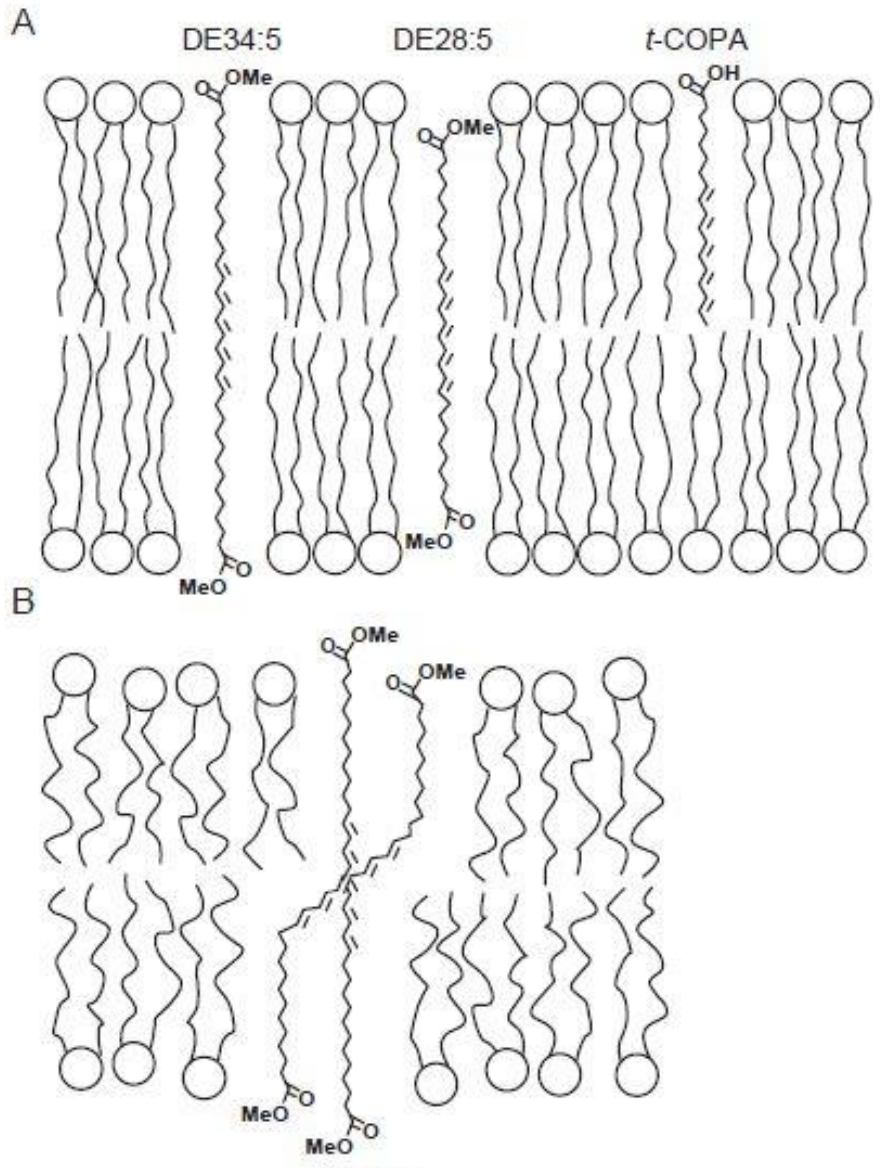

DE34:5

\begin{abstract}
Scheme 1. Pictorial representation (approximately to scale) of the membranespanning biamphiphilic pentaene diesters DE28:5 and DE34:5, as well as of the amphiphilic pentaenoic acid $t$-COPA, in a DMPC lipid bilayer. The long polyene bolaamphiphiles tend to be aligned with the ordered acyl chains in the bilayer gel phase (A). In contrast, large conformational fluctuations of probe molecules may be possible in the fluid phase (B), illustrated here with a $\sim 50^{\circ}$ oscillation of the polyene group of the diester DE34:5.
\end{abstract}

The distance between the $\alpha, \omega$-methoxycarbonyl groups of the transmembrane probes was designed as to match approximately the thickness of typical phospholipid bilayers. The steady-state and time-resolved fluorescence anisotropy of the biamphiphilic polyenes in large unilamellar vesicles (LUVs) made up from 


\section{1,2-dimiristoyl-sn-glycero-3-phosphatidylcholine \\ (DMPC) \\ or \\ 1,2-dipalmitoyl-sn-glycero-3-}

phosphatidylcholine (DPPC) were studied here at several temperatures, and compared with that of the single-headed amphiphilic probe t-COPA. In this way, the rotational diffusion constants of the bolapolyenes in the lipid bilayer and the anchoring effect of the symmetric polar heads on the lipid dynamics could be determined.

\section{Materials and methods}

\subsection{Materials}

Fluorescent biamphiphilic diesters DE28:5, DE34:5 and other polyene analogs were synthesized and purified as described in detail elsewhere [25]. The monopolar amphiphilic pentaenoic acid t-COPA was synthesized as described before [26]. Synthetic phospholipids DMPC and DPPC were from Sigma (St. Louis, Mo., USA) and were used as received.

\subsection{Sample preparation}

Large unilamellar vesicles (LUVs) of $\sim 100 \mathrm{~nm}$ diameter containing the transmembrane fluorescent bolapolyenes were prepared by extrusion. Briefly, $10 \mu \mathrm{L}$ of a $10-4 \mathrm{M}$ chloroform solution of the bolapolyene were mixed with $100 \mu \mathrm{L}$ of a solution of a synthetic phospholipid in the same solvent (ca. $1 \mathrm{mg} / \mathrm{mL}$ ). The mixture was first dried under a stream of Ar, and then vacuum dried with gentle warming to eliminate the remaining traces of the solvent. The dry film was hydrated with $200-300 \mu \mathrm{L}$ of buffer solution $(5 \mathrm{mM}$ phosphate-saline, $\mathrm{pH} 7)$ or deionized water, with gentle vortexing and warming above the lipid thermal transition temperature. The multilamellar vesicle suspension obtained in this way was passed 10 times through calibrated filters (nominal pore size $0.1 \mu \mathrm{m}$ ) using an Avanti Mini-Extruder 61000 (Avanti Polar Lipids Inc., AL, USA) fitted with a heating block. Total lipid concentration was determined as phosphomolybdate by established methods. In the case of DE28:5 the LUVs obtained in this form contained a probe:lipid molar ratio in the range $1: 300-1: 500$, as determined from absorption measurements; the incorporation of DE34:5 was, however, somewhat more erratic, due probab by to the limited solubility of the bolapolyenes in these lipids. The spectroscopic measurements were carried out immediately after sample preparation.

\subsection{Methods}

Absorption measurements were run on a Varian Cary 3E spectrophotometer, fitted with a Peltier temperature controller. Steady-state corrected fluorescence intensity and anisotropy (rs) measurements were recorded in a SLM 8000D spectrofluorimeter [23]. Timeresolved intensity and polarization measurements were performed with the time-correlated single-photon counting technique. Excitation $(\lambda e x c=310-315 \mathrm{~nm})$ was produced by third harmonic conversion of $1 \mathrm{ps}$ pulses from a Ti:saphire mode-locked laser (Tsunami, Spectra-Physics, MountainView, CA, USA) pumped with a Nd:YVO4 laser (5 W, Millennia, Spectra-Physics). The linear polarization of the laser pulses was selected with a Berek compensator (model 5540, New Focus, USA) and a Glan-Taylor prism. The fluorescence from samples placed in thermostated $3 \times 3 \mathrm{~mm}$ cuvettes, collected in the horizontal plane and at $90^{\circ}$ to the excitation beam, was focused on a monochromator ( $\mathrm{f}=100 \mathrm{~mm}, 8 \mathrm{~nm}$ bandwidth) through cut-off filters, a motorized Glan-Taylor polarizer and a quartz depolarizer (Acton Research Co.), and finally detected with a R1564U-06 microchannel plate photomultiplier (Hamamatsu Photonics Co.). The detector pulses were amplified ( $\times 50$, Phillips Scientific, USA, model 6954$)$ and fed to counting electronics. Data were stored in $2 \mathrm{~K}$ channels with 6-13.1ps/channel resolution up to 106 total counts. The instrument response function, recorded from exciting laser pulses dispersed by pure water, was $\sim 70 \mathrm{ps}$. The decay of the total fluorescence intensity (Im) was recorded with the emission polarizer set at the 'magic angle' $\mathrm{m}=54.7^{\circ}$, relative to the plane of the vertically polarized excitation beam. The two polarized components of the fluorescence, parallel (Ivv) and perpendicular (Ivh) to the polarization plane of the excitation beam, were recorded sequentially in time steps of $2 \mathrm{~min}$.

The experimental anisotropy is given by:

$$
R(t)=\left[I_{\mathrm{vv}}(t)-G I_{\mathrm{vh}}(t)\right] /\left[I_{\mathrm{vv}}(t)+2 G I_{\mathrm{vh}}(t)\right]
$$


where $\mathrm{G}$ is a scaling factor that accounts for differences in counting times and detector efficiency for the two polarized intensities. The $\mathrm{G}$ factor was determined by normalizing the total count number under the $\mathrm{R}(\mathrm{t})$ experimental trace to match the value of the steady-state anisotropy of the sample separately measured [27], and varied in the 0.99-1.02 range.

2.4. Analysis of fluorescence intensity and anisotropy decay times

The decay of the fluorescence intensity I was best fitted to the sum of two exponential functions, $\mathrm{i}(\mathrm{t})=\mathrm{a} 1 \exp (-\mathrm{t} / \tau 1)+\mathrm{a} 2$ $\exp (-t / \tau 2)$, by iterative convolution, using non-linear leastsquares methods from the Globals Unlimited (Urbana, ILL) generalpurpose program [28]. The anisotropy decay function $\mathrm{r}(\mathrm{t})$ was computed by simultaneous analysis of the two polarized components of the emission, introducing the lifetime data as fixed parameters and using the same routines. The anisotropy decay corresponding to the lipid bilayer fluid phase was fitted to a three-exponential numerical expression:

$$
r(t)=\left(r_{0}-r_{\infty}\right)\left[\sum_{i=1}^{3} \beta_{i} \exp \left(-t / \phi_{i}\right)\right]+r_{\infty}
$$

in which $\mathrm{r} 0$ is the theoretical anisotropy of the emitting polyene (i.e., the anisotropy value in absence of any depolarizing process), r $\infty$ is the residual anisotropy at $\mathrm{t} \rightarrow \infty, \phi \mathrm{i}$ are correlation times, and $\beta \mathrm{i}$ the normalized amplitudes: $\Sigma \beta \mathrm{i}=1$. The r0 value, which is a spectroscopic property of the conjugated pentaene system, was taken as that determined experimentally for the amphiphilic probe $t$ COPA (0.385 \pm 0.005$)$ [23] and introduced as a fixed parameter in the fitting routines. The quality of lifetime and anisotropy fits was determined from the $\chi^{2}$ values and visual inspection of the weighted residuals distribution. A simpler two-exponential function was clearly inadequate to fit the experimental anisotropy decay, as shown below. At temperatures corresponding to the gel lipid-phase, the fluorescence anisotropy of the bolapolyenes remained constant along the emission time-window, apart from a small timedependent segment observed in the first nanoseconds of the $\mathrm{r}(\mathrm{t})$ traces. The subnanosecond time-constant of this decay could only be estimated, due to its small weight and the overlap with a vertically polarized residual scattering component $(<5 \%)$. The ordering effect of the bilayer on a fluorescent probe is frequently assessed from the value of the probe order parameter Sp which, after several simplifying assumptions [18-20,23] can be obtained from the expression:

$$
S_{\mathrm{p}}=\left(\frac{r_{\infty}}{r_{0}}\right)^{1 / 2}
$$

\section{Results}

3.1. Absorption and emission properties of the bipolar probes Bolapolyenes containing two free carboxylic headgroups are only slightly soluble $(<10-6 \mathrm{M})$ in solvents as methanol, ethanol, chloroform or dimethylformamide. This also limits the amount of these compounds that can be incorporated into lipid bilayers of DMPC or DPPC. However, if the carboxylic groups are replaced by the corresponding methyl ester groups, the solubility increases considerably, and no apparent changes were observed in the absorption, emission and lifetime parameters (data not shown). Therefore, the spectral and dynamic characterization of the bolapolyenes, both in pure solvents and in lipid bilayers, was carried out here with the more soluble methyl ester derivatives. Representative absorption and fluorescence spectra of the conjugated pentaene diester DE28:5, incorporated into LUVs of DMPC (ca. 100nm diametre, 1:400 probe / lipid molar ratio) are shown in Fig. 1; Table 1 lists absorption and emission properties of this compound together with those of bolapolyenes containing 4 and 6 conjugated double bonds, for comparison. At the resolution used here, the structured absorption spectra of the tetraene and pentaene biamphiphiles are virtually identical to those of the corresponding parinaric and t-COPA fatty acids mentioned above [22,23], and are not affected by the length of the hydrophobic chain connecting the polyene system with the two methoxycarbonyl headgroups (from C8 to C11). In addition, the absorption spectra recorded in bilayers are the same as those in fluid solvents, apart from the expected small shifts $(\leq 2 \mathrm{~nm})$ due to the effect of solvent polarizability $[22,23]$. This excludes the presence of bolapolyene aggregates within the bilayer. The fluorescence emission of the bolapolyenes, both in solution and in lipid bilayers, consists of a broad band (Fig. 1) with a large Stokes shift $(\sim 7000 \mathrm{~cm}-1)$. As in the case of the absorption spectra, the shape 
and position of the emission band are not very different from those of the corresponding fatty acid, and are not affected by the length of the saturated polymethylene spacer or by esterification of the carboxylic acid head groups. The emission band position is not very sensitive to solvent polarizability, in contrast with what is observed in the absorption transition. The fluorescence quantum yield of DE28:5 and DE34:5 bolapolyenes incorporated into lipid vesicles is $\Phi F=0.14 \pm 0.03$ (DMPC, $25^{\circ} \mathrm{C}$ ), coincident with that of the pentaene amphiphilic fatty acid t-COPA. Moreover, in common also with the monopolar compound, the fluorescence yield of the transmembrane bolapolyenes is only slightly affected by temperature in the $15-35^{\circ} \mathrm{C}$ range.

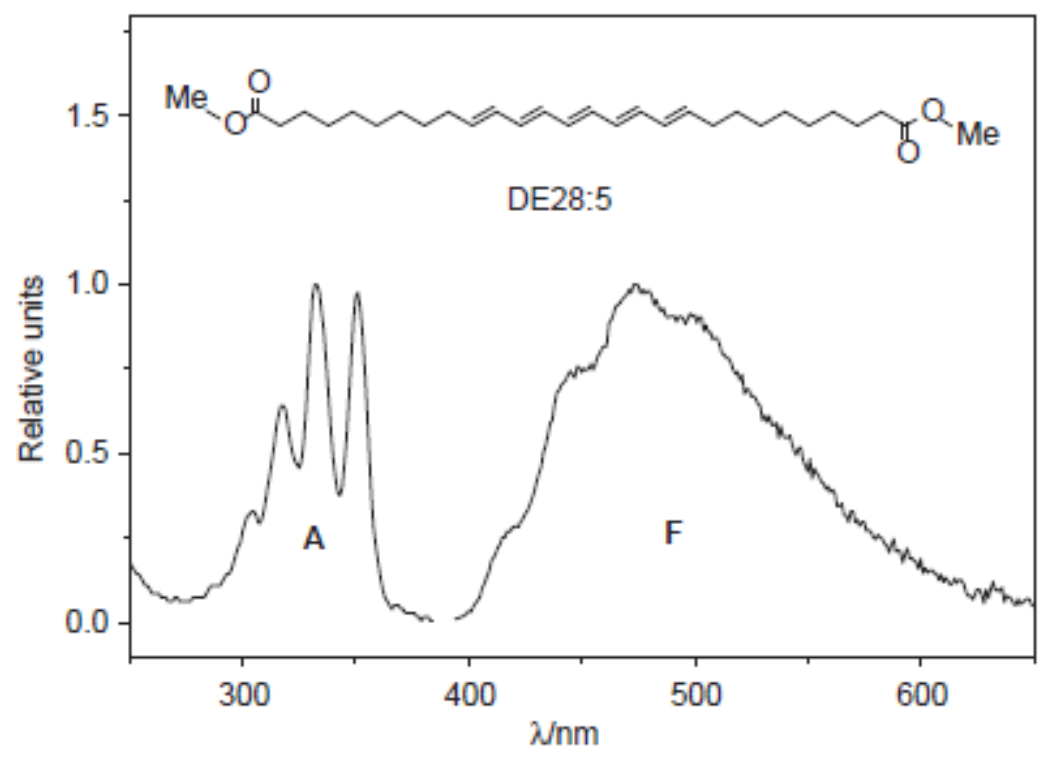

Fig. 1. Absorption (A) and corrected fluorescence (F) spectra of the pentaene diester DE28:5 $4 \times 10^{-7} \mathrm{M}$ in LUV vesicles of DMPC, $28^{\circ} \mathrm{C}$. $\lambda_{\text {exc }} 333 \pm 2 \mathrm{~nm}$; probe:lipid $\sim 1: 400$.

Table 1

Absorption and fluorescence properties of biamphiphilic diesters

\begin{tabular}{lllll}
\hline Sample $^{\mathrm{a}}$ & Solvent & $\lambda_{\text {abs }}(\mathrm{nm}, \pm 0.5)$ & $\varepsilon\left(\mathrm{M}^{-1} \mathrm{~cm}^{-1}, \pm 2000\right)$ & $\lambda_{\mathrm{f}}{ }^{\mathrm{b}}(\mathrm{nm}, \pm 2)$ \\
\hline $\mathrm{DE} 32: 4$ & $\mathrm{CHCl}_{3}$ & 320.6 & 65000 & 408 \\
& & 306 & 73000 & \\
& & 293 & 49000 & \\
DE28:5 & $\mathrm{CHCl}_{3}$ & 353 & 92000 & 474 \\
& & 335 & 95000 & \\
& & 320 & 60000 & 539 \\
DE30:6 & $\mathrm{CHCl}_{3}$ & 383 & 124000 & \\
& & 362 & 120000 & \\
& & 344 & 71000 & \\
DE28:5 & $\mathrm{LUVs}^{\mathrm{c}}$ & 351 & & \\
& & 333 & & \\
& & 318 & & \\
\hline
\end{tabular}

Absorption and fluorescence properties of representative transmembrane biamphiphilic all-(E)-polyene diesters with 4,5 and 6 conjugated double bonds.

${ }^{a}$ DEm: $n, m$ total number of carbon atoms, $n$ total number of double bonds (Scheme 1).

${ }^{\mathrm{b}}$ Wavelength maximum in the corrected fluorescence spectra.

${ }^{c}$ Large unilamellar lipid vesicles of DMPC in gel phase $\left(15^{\circ} \mathrm{C}\right)$. 


\subsection{Fluorescence polarization}

The fluorescence of the pentaenes DE28:5 and DE34:5 embedded in gel-phase lipid vesicles (LUVs of DMPC or DPPC, $10^{\circ}$ ) is strongly polarized, with average values of the steady-state anisotropy (rs) in the $0.35-0.36$ range, as expected for the parallel orientation of the absorption and emission transition moments of the conjugated double bond system [23,29]. When the temperature is increased up to that of the bilayer liquid-crystal phase, the anisotropy decreases down to a very low value, indicating a large mobility of the polyene. The anisotropy change as a function of temperature is shown in Fig. 2, together with that of the amphiphilic probe t-COPA in similar conditions, for comparison. A sharp drop in the rs value occurs at the main lipid phase transition temperature (23.5 and $41^{\circ} \mathrm{C}$ for DMPC and DPPC, respectively), indicating that polyene mobility accurately follows the changes in order and dynamics of the bilayer. The anisotropy changes of the monopolar and bipolar probes are very similar, the only important difference being the somewhat higher rs value of the DE28:5 analog in the fluid phase.

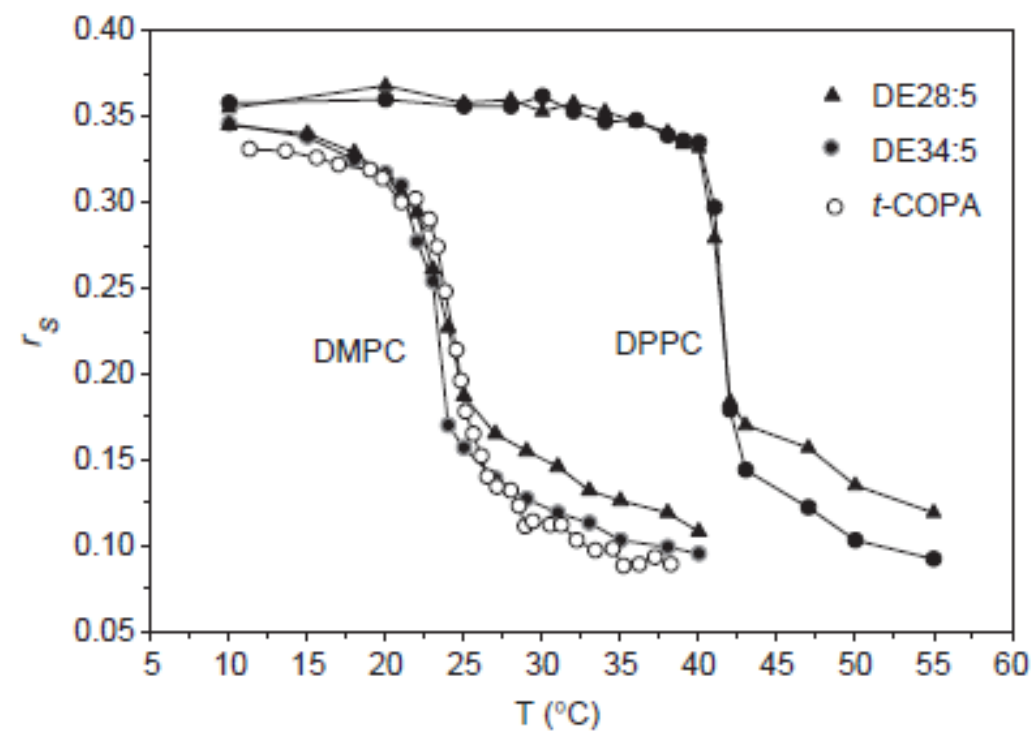

Fig. 2. Steady-state fluorescence anisotropy $\left(r_{\mathrm{S}}\right)$ of the pentaene diesters DE28:5 and DE34:5 in LUV vesicles of DMPC or DPPC as a function of temperature. $\lambda_{\text {exc }} 333 \pm 2 \mathrm{~nm} ; \lambda_{\mathrm{em}} 475 \pm 16 \mathrm{~nm}$. The data for the amphiphilic pentaenoic acid $t$-COPA in DMPC vesicles are also shown for comparison (adapted from Ref. [23]).

3.3. Time-resolved fluorescence intensity and anisotropy

The decay of the fluorescence intensity of the biamphiphilic polyenes DE28:5 and DE34:5 was recorded in LUVs of DMPC at temperatures corresponding to the gel and fluid phases. The emission traces were well fitted to a two-exponential function (Table 2), with lifetime values somewhat different from those of the amphiphilic pentaene t-COPA [23]. The slowest component of the emission decay accounts for $\sim 98 \%$ of the total stationary fluorescence intensity, and shows only a modest change as a function of the lipid thermal phase, that explains the corresponding small temperature dependence of the quantum yield. The relatively large value of this dominant lifetime is consistent with the forbidden character of the condensed-phase fluorescence transition in these polyenes (v. infra) [30,31]. The decay of the fluorescence anisotropy of the bolapolyenes was also recorded in LUV vesicles of DMPC at temperatures corresponding to the gel and fluid lipid phase. Representative decay traces for DE28:5 are shown in Fig. 3, where it can be observed that the anisotropy value in the gel phase $\left(12^{\circ} \mathrm{C}\right)$ remains unchanged along the fluorescence lifetime of the pentaene chromophore. The small decay component in the first ns could only be estimated (see Methods). In the fluid, liquidcrystalline phase, the bipolar polyene shows a large mobility, which is reflected by a complex decay of the anisotropy (Table 3 ) down to a small residual value ( $r \infty)$. The decay of the longer probe DE34:5 was also very similar. In both cases, the time-dependent part of the experimental traces extends over almost two orders of magnitude (from fractions to tens of nanoseconds) and required a three-exponential function to be fitted with good statistics. Within the present accuracy, the anisotropy decay was the same for the 
two lifetime components (non-associative model). The data corresponding to the monopolar probe t-COPA, recorded at $31^{\circ} \mathrm{C}$ in similar lipid vesicles [23], were also included in Table 3 for comparison. Although the excitation pulse length used in Ref. [23] limited the timeresolution of the t-COPA experiments, it can be seen that the value of the correlation time $\phi 3$ is a factor of two slower in the case of the bolapolyenes.

Table 2

Lifetime parameters of the pentaene diesters DE28:5 and DE34:5, and of the pentaene fatty acid $t$-COPA, in LUV vesicles of DMPC

\begin{tabular}{lllllll}
\hline Sample & $\begin{array}{l}T \\
\left({ }^{\circ} \mathrm{C}\right)\end{array}$ & $\begin{array}{l}a_{1} \\
( \pm 0.02)\end{array}$ & $\begin{array}{l}\tau_{1} \\
(\mathrm{~ns}, \pm 0.04)\end{array}$ & $\begin{array}{l}a_{2} \\
( \pm 0.04)\end{array}$ & $\begin{array}{l}\tau_{2} \\
(\mathrm{~ns}, \pm 0.2)\end{array}$ & $\begin{array}{l}\langle\tau\rangle^{\mathrm{a}} \\
(\mathrm{ns}, \pm 0.5)\end{array}$ \\
\hline DE28:5 & 12 & 0.25 & 2.02 & 0.75 & 20.7 & 20.2 \\
& 35 & 0.22 & 1.35 & 0.78 & 18.4 & 18.0 \\
DE34:5 & 12 & 0.33 & 1.48 & 0.67 & 20.4 & 19.7 \\
& 35 & 0.26 & 1.25 & 0.74 & 17.6 & 17.3 \\
$t$-COPA & 30 & 0.16 & 2.3 & 0.84 & 19.1 & 18.7 \\
\hline
\end{tabular}

${ }^{a}$ Average lifetime $\langle\tau\rangle=\Sigma a_{i} \tau_{i}^{2} / \Sigma a_{i} \tau_{i}$.

b From Ref. [23].
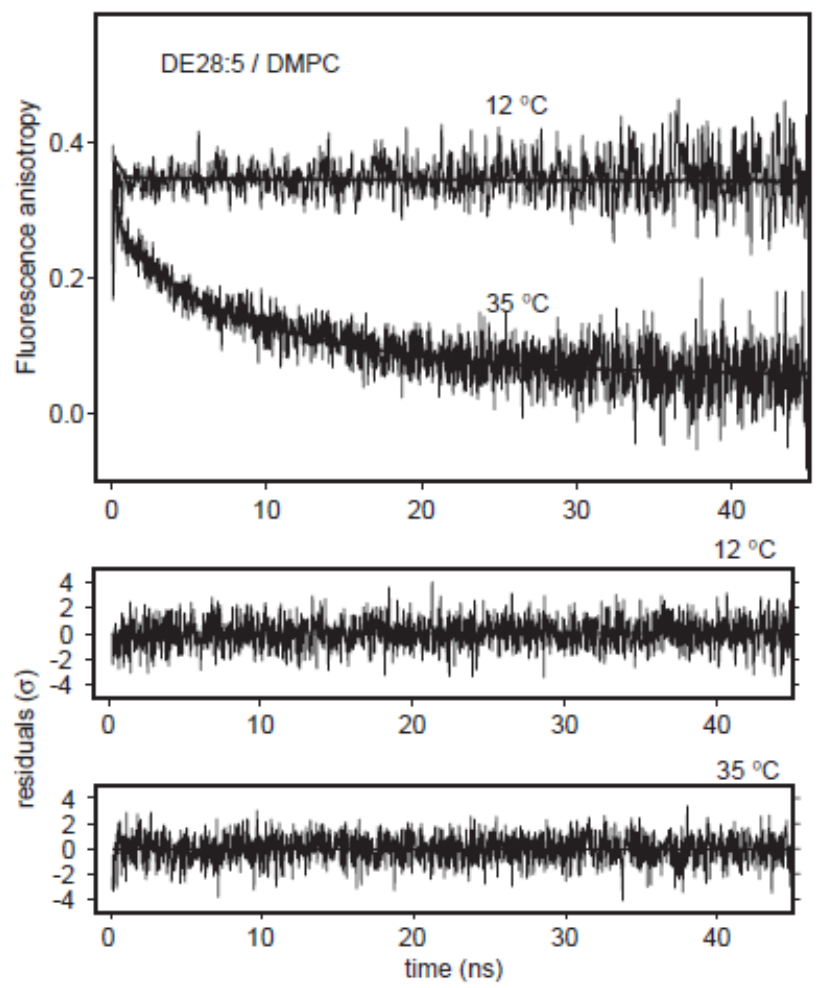

Fig. 3. Time-resolved fluorescence anisotropy of the pentaene diester DE28:5 in LUV vesicles of DMPC, in gel $\left(12^{\circ} \mathrm{C}\right)$ and fluid $\left(35^{\circ} \mathrm{C}\right)$ lipid phase. $\lambda_{\text {exc }}$ $310 \mathrm{~nm} ; \lambda_{\mathrm{em}} 510 \pm 8 \mathrm{~nm}$. The parameters of the corresponding fitting functions (smooth line) are listed in Table 3.

\section{Discussion}

4.1. Transmembrane orientation of the biamphiphilic polyenes in lipid bilayers

The distance between the two opposite ester groups in the fully stretched conformation of the bolapolyenes DE28:5 and DE34:5 is 34 and $42 \AA$, respectively, as estimated from standard bond lengths and angles. These values are very similar to those obtained from more elaborated techniques (MOPAC 93 energy minimization) [32]. The length of the linear chain was designed as to span the 
thickness of typical fluid-phase bilayers (for example, the head-to-head distance is $\sim 36 \AA$ in a DMPC fluidphase bilayer [33]). Therefore, a plausible orientation of the bolapolyenes in DMPC or DPPC spherical vesicles would be spanning the bilayer, with the conjugated pentaene system aligned with the phospholipid acyl chains and located in the middle, hydrocarbon-like part of the membrane, as pictured in Scheme 1. The large change in the steady-state anisotropy of the polyenes occurring at the lipid thermal transition temperature (Fig. 2) is an indirect indication of this specific orientation. Such a large decrease of fluorescence polarization would be unlikely if the polyene group were located in a peripheral orientation at or near the membrane surface. Moreover, a membrane-spanning orientation is also consistent with the observed blue shift of the polyene absorption band in the bilayer, compared with a chloroform solution (Table 1), which is indicative of a lower polarizability microenvironment in the first case [23]. A more direct evidence of the transmembrane alignment of the bolapolyenes can be obtained by polarized two-photon fluorescence microscopy techniques [34]. In this way it could be shown that the fluorescence transition moment of the polyene DE24:5 in 1palmitoyl-2-oleoyl-sn-glycero-3-phosphatidylcholine giant vesicles is, on the average, aligned parallel to the phospholipid acyl chains [25a]. This should be also the orientation of the polyene group, since the electronic transition moment is nearly parallel to the polyene long axis [29,35]. Moreover, these experiments also excluded a bent conformation in which both head groups of the polyene are localized on the same face of the bilayer.

\begin{tabular}{|c|c|c|c|c|c|c|c|c|c|}
\hline Sample & $T\left({ }^{\circ} \mathrm{C}\right)$ & $\beta_{1}( \pm 0.05)$ & $\phi_{1}(\mathrm{~ns}, \pm 0.1)$ & $\beta_{2}( \pm 0.02)$ & $\phi_{2}(\mathrm{~ns}, \pm 0.2)$ & $\beta_{3}( \pm 0.04)$ & $\phi_{3}(\mathrm{~ns}, \pm 0.03)$ & $r_{\infty}( \pm 0.01)$ & $r_{s}( \pm 0.004)$ \\
\hline \multirow[t]{2}{*}{ DE28:5 } & 12 & 0.04 & $\sim 0.40$ & & & & & 0.35 & 0.345 \\
\hline & 35 & 0.33 & 0.14 & 0.13 & 2.1 & 0.54 & 11.5 & 0.05 & 0.125 \\
\hline \multirow[t]{2}{*}{ DE34:5 } & 12 & 0.03 & $\sim 0.30$ & & & & & 0.34 & 0.346 \\
\hline & 35 & 0.29 & 0.14 & 0.14 & 0.9 & 0.57 & 10.6 & 0.03 & 0.112 \\
\hline$t=\mathrm{COPA}^{\mathrm{a}}$ & 31 & 0.39 & 0.2 & 0.27 & 1.3 & 0.34 & 5.6 & 0.072 & 0.110 \\
\hline
\end{tabular}

${ }^{a}$ From Ref. [23].

\subsection{Photophysics of the polyene biamphiphilic probes}

The spectral features observed in the 200-700nm range in low viscosity solvents are, of course, due to the central polyene system, because the long saturated polymethylene chains prevent any electronic interaction between pentaene and methoxycarbonyl groups. Therefore, the strong first absorption band is assigned to the allowed transition to the second excited singlet state, $1 \mathrm{Bu} \leftarrow 1 \mathrm{Ag}$, in a symmetric planar pentaene [29-31]. It is well known that this transition is sensitive to solvent polarizability, shifting to lower energies as the solvent polarizability increases [23,29]. This may explain the small absorption shifts mentioned above (Table 1), as well as that taking place $(\sim 2 \mathrm{~nm}$, data not shown) when the bilayer changes from fluid to the high-density gel phase (higher polarizability). The fluorescence emission arises from the $21 \mathrm{Ag} \rightarrow 11 \mathrm{Ag}$ forbidden transition [29-31], which is insensitive to solvent polarizability. The absorption and emission transition moment orientation are coincident and nearly parallel to the long axis of the polyene system $[20,35]$.

\subsection{Dynamics of bolaamphiphiles in the bilayer}

The steady-state anisotropy value of DE28:5 and DE34:5 in a gel-phase lipid bilayer is close to that of a completely immobilized pentaene (0.385) [23], indicating an extremely rigid conformation of the ester molecules in these conditions. In contrast, the low anisotropy value in the liquid-crystal phase corresponds to a highly mobile fluorophore. Since the emitting group is anchored to both sides of the bilayer by the polar ester heads, its large-amplitude reorientational motions must be accompanied by important lateral and transverse shifts of the chains and headgroups of the bolapolyene (Scheme 1). The higher rs values of DE28:5, compared to those of DE34:5 (Fig. 2), in the lipid fluid-phase originate from a somewhat more restricted polyene mobility of the shorter-chain compound (v. infra). This small difference is not strongly sensitive to hydrophobic mismatch between DE28:5 bolapolyene length $(\sim 34 \AA)$ and bilayer thickness $(\sim 36 \AA)$, which should increase in the thicker DPPC bilayer ( 41 of the pentaene amphiphilic probe t-COPAwere also included in Fig. 2 for comparison. The similarity between the steady-state values of mono- and bipolar (DE34:5) polyenes in fluid-phase bilayers is only accidental, as can be seen from the time-resolved data. In fact, the depolarization kinetics of the bolapolyene contains a relaxation time $(\phi 3)$ of $\sim 11 \mathrm{~ns}$ (Table 3$)$, which is a factor of two slower than that of the monopolar probe. On the other hand, the correlation times $\phi 1$ and $\phi 2$ are similar to those of t-COPA and, most likely, originate in conformational fluctuations of the saturated hydrocarbon chain extending over one or a few single bonds (e.g., trans/gauche isomerization). These motions are not expected to be greatly different in the monopolar and bipolar compounds. 
According to that, the correlation time $\phi 3$ may be assigned to the tilting motions of the complete molecule, since the overall reorientation of the bolapolyene is expected to be substantially slower than that of the monopolar compound. The similarity between steady-state anisotropy values noted above arises from the compensation of the different correlation times, fluorescence lifetimes and residual anisotropy of the two polyenes. The multiple correlation times recorded for the bolapolyenes in lipid bilayers span a time range from hundreds of picoseconds to tens of ns. The interpretation of this complex dynamics in terms of rotational diffusion coefficients requires first assigning a physical model to each depolarizing process. In the 'compound motion' model [36], the reorientational motion of a fluorescent probe in a bilayer is approximated by the superposition of fast local motions of the probe in a lipid 'cage', and by a slower rotation of the cage within the bilayer. The probe motion in the first case is analytically approximated by the wobble-in-cone model, in which the molecule diffuses freely confined to a conical space $[15,16]$. The slower motion of the cage is represented by a Brownian Rotational Diffusion (BRD) taking place in an orienting potential [17].As would be expected, fitting the resulting complex analytical expression to the probe anisotropy decay requires the optimization of five adjustable parameters. A meaningful application of this model is only possible when the experimental anisotropy decay traces show a very high signal/noise ratio, which is not the case here. Instead, the dynamics of the bolapolyenes and of their immediate lipid environment would be interpreted by comparing the angular motions of the mono- and biamphiphilic pentaenes. This can be done in a simple way by assuming that the bipolar probe diffuses freely in a Gaussian-shaped angular potential [37-39], as in the case of tCOPA [23]. In this approach, the average relaxation time $\langle\phi\rangle$ can be obtained from the area under the multiexponential anisotropy decay curve, Eq. (2), by the expression:

$$
\langle\phi\rangle=\int_{0}^{\infty}\left[\left(r(t)-r_{\infty}\right) /\left(r_{0}-r_{\infty}\right)\right] \mathrm{d} t=\sum_{1}^{n} \beta_{i} \phi_{i} .
$$

The $\langle\phi>$ value is related to the average rotational diffusion constant $\mathrm{D} \perp$ around an axis perpendicular to the long molecular axis of the emitting pentaene group by the expression:

$$
\langle\phi\rangle=\frac{\sigma_{\mathrm{G}}}{D_{\perp}}
$$

in which $\sigma \mathrm{G}$ is a parameter that depends on the Gaussian-shaped potential restricting the probe angular oscillation. Numerical values of $\sigma \mathrm{G}$ as a function of the $\mathrm{r} \infty / \mathrm{r} 0$ experimental value can be obtained from a polynomial approximation [23] to the data of Kinoshita et al. [39], as follows:

$$
\sigma_{\mathrm{G}}=0.1674-0.1066\left(r_{\infty} / r_{0}\right)-0.062\left(r_{\infty} / r_{0}\right)^{2}
$$

Finally, the angle amplitude of the Gaussian 'cone' $\theta \mathrm{G}$ can be found from the experimental value of the order parameter Sp [38]. 
Table 4

Diffusion constants, order parameters and cone semi-angle amplitude of the biamphiphilic diesters ${ }^{\mathrm{a}}$

\begin{tabular}{llllll}
\hline Sample & $T\left({ }^{\circ} \mathrm{C}\right)$ & $\langle\phi\rangle^{\mathrm{b}}(\mathrm{ns}, \pm 0.2)$ & $D_{\perp}\left(\mathrm{rad}^{2} \mathrm{~ns}^{-1}, \pm 0.002\right)$ & $S_{\mathrm{P}}{ }^{\mathrm{c}}$ & $\theta_{\mathrm{G}}{ }^{\mathrm{c}}$ \\
\hline DE28:5 & 35 & 6.9 & 0.022 & 0.36 & $50^{\circ}$ \\
DE34:5 & 35 & 6.2 & 0.026 & 0.28 & $56^{\circ}$ \\
$t$-COPA & 31 & 2.3 & 0.063 & 0.43 & $44^{\circ}$ \\
\hline
\end{tabular}

${ }^{\text {a }}$ Average rotational diffusion constant $D_{\perp}$, order parameter $S_{\mathrm{p}}$ and cone semiangle amplitude $\theta_{\mathrm{G}}$ of the diesters DE28:5 and DE34:5 in DMPC LUV vesicles, as computed from the wobbling-in-a Gaussian potential model.

${ }^{\mathrm{b}}$ Average correlation time $\langle\phi\rangle=\sum_{1}^{3} \beta_{i} \phi_{i}$.

c $20 \%$ estimated uncertainty.

${ }^{d}$ From Ref. [23].

The experimental and derived quantities are listed in Table 4, and clearly indicate that: i) the rate of reorientationalmotion of the transmembrane biamphiphiles as measured by the $\mathrm{D} \perp$ value is only a $30 \%$ of that of $\mathrm{t}-\mathrm{COPA}$; and ii) the equilibriumorientational distribution of the bipolar probes is highly disordered, with cone semi-angles of 50-56 $6^{\circ}$, similar to that of the monopolar pentaene. Since the conjugated pentaene group is not flexible, the large angular oscillation of the bipolar probes must be coupled to very fast

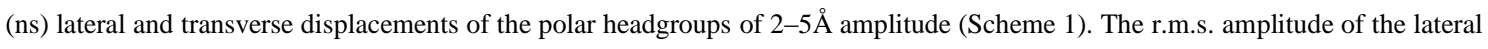
and verticalmotions of the polar headgroups, estimated in a relatively model-independent way from the order parameter expression, also yields similar amplitude values. These simple computations indicate that the fluorescent bolapolyenes highfrequency oscillatory motions take place in a very low friction microenvironment ('slip' regime). Moreover, it is shown that the polymethylene chains that anchor the polyene group to the two opposite bilayer surfaces largely decrease the wobbling rate of the emitting group, but do not restrict the angular amplitude of these motions.

The novel technique of single-molecule anisotropy imaging may yield a more direct measurement of $\mathrm{D} \perp$ values for phospholipids tagged with fluorescent dyes. In this way a value of $\mathrm{D} \perp$ of $0.07 \mathrm{rad} 2 \mathrm{~ns}-1$ has been found [40] for the diffusion constant of a tetramethylrhodamine dye tethered to the polar head of a phospholipid molecule dissolved in a bilayer of POPC at $22^{\circ} \mathrm{C}$. The coincidence of this value with that of t-COPA (Table 4) is likely to be accidental, because the depolarizing motions are different in the two molecules. Theoretical simulations of the depolarizing motions of bolalipids are not yet available, as far as we are aware. However, detailed simulations have been carried out on the ns rotational diffusion of an amphiphilic probe immersed in a lipid bilayer, using Brownian dynamics methods [41] in which an extended computational time-range (hundreds of ns) compensates the loss of molecular detail. These simulations reproduce qualitatively the kinetic features of the monopolar polyene reorientation mentioned above. On the other hand, Molecular Dynamics (MD) simulations, which should be capable, in principle, of yielding an accurate description of probe dynamics down to atom resolution, still are limited by a reduced computation time range [2]. In a recent study of this kind [42], the rotational mobility of individual phospholipid molecules of a DMPC bilayer was analyzed along $3 \mathrm{~ns}$, giving a value of $\mathrm{D} \perp=25 \mathrm{rad} 2 \mathrm{~ns}-1$ for the rotational diffusion constant of each of the two $\mathrm{C} 14$ lipid chains, at large variance with the experimental t-COPA value of $0.07 \mathrm{rad} 2 \mathrm{~ns}-1$ (Table 4). Moreover, the predicted value of $\mathrm{D} \perp$ for the polar group rotation was $2.2 \mathrm{rad} 2 \mathrm{~ns}-1$, which also departs considerably from the experimental one as measured by single molecule imaging [40]. The source of these discrepancies is thought to be the reduced time-scale of the MD simulations [2].

\section{Conclusions}

The average nanosecond rotational diffusion constant of transmembrane bolapolyenes in fluid bilayers of DMPC has been found to be only $1 / 3$ of that of the parent monopolar polyene probe, due most likely to the anchoring effect of the two polar headgroups; in contrast, the fast localized dynamics of the long polymethylene connectors is very similar in both cases. In addition, a large angular amplitude of the rotational motions of the polyene group $\left(\theta\right.$ half-cone $\approx 50^{\circ}$ ) was recorded, indicating weak frictional forces and lack of orientational restrictions of the bilayer midplane for the partially flexible bolaamphiphile structure. As a result of that, lateral excursions of the polar headgroups of the bolapolyenes, parallel and perpendicular to the bilayer surface and with 2-5Å amplitude, must also occur in the ns time-range, to allow for the large angular mobility of the polyene emitting group. The experiments 
reported here also indicate that the microscopic fluid properties of a bolalipid monolayer membrane may not be substantially different from those of ordinary lipid bilayers.

Acknowledgements

We are grateful to Dr. N. Michaylov and R. Fernández for their valuable help in data recording, and to J. Delgado for sample purification. Work financed by the Ministerio de Ciencia y Tecnología of Spain, Grants BQU2000-1500 and BQU2003-04413.

References

[1] S. Tristram-Nagle, J.F. Nagle, Lipid bilayers: thermodynamics, structure, fluctuations and interactions, Chem. Phys. Lipids 127 (2004) 3-14.

[2] H.L. Scott, Modeling the lipid component of membranes, Curr. Opin. Struck. Biol. 12 (2002) 495-502.

[3] J. Seelig, A. Seelig, Lipid conformation in model membranes and biological membranes, Quart. Rev. Biophys. 13 (1980) 19-61.

[4] L.B. Silver, The Physical Chemistry of Membranes, Allen and Unwin Inc., Mass., USA, 1985.

[5] M. Bloom, E. Evans, O.G. Mouritsen, Physical properties of the fluid lipidbilayer component of cell membranes: a perspective, Quart. Rev. Biophys. 24 (1991) 293-397.

[6] M. De Rosa, A. Gambacorta, The lipids of archaebacteria, Prog. Lipid Res. 27 (1988) 153-175.

[7] M. Kates, D.J. Kushner, A.T. Matheson, The Biochemistry of Archae [Archaebacteria], Elsevier, Amsterdam, 1993, pp. 125134.

[8] J.-H. Fuhrhop, T.Wang, Bolaamphiphiles, Chem. Rev. 104 (2004) 2901-2937.

[9] A. Gliozzi, A. Relina, P.L.-J. Chong, Structure and permeability properties of biomimetic membranes of bolaform archaeal tetraether lipids, J. Membr. Sci. 206 (2002) 131-147.

[10] W.L.C. Vaz, D. Hallmann, R.M. Clegg, A. Gambacorta, M. De Rosa, A comparison of the translational diffusion of a normal and a membranespanning lipid in L $\alpha$ phase 1-palmitoyl-2-oleoylphosphatidylcholine bilayers, Eur. Biophys. J. 12 (1985) 19-24.

[11] L.B.Å. Johansson, G.Q. Lindblom, Orientation and mobility of molecules in membranes studied by polarized light spectroscopy, Quart. Rev. Biophys. 13 (1980) 63-118.

[12] W.H. Sawyer, Fluorescence spectroscopy in the study of membrane fluidity: model membrane systems, in: R.C. Aloia, C.C. Curtain, L.M. Gordon (Eds.), Methods for Studying Membrane Fluidity, vol. 1, Alan R. Liss Inc., New York, 1988, pp. $161-191$.

[13] C.D. Stubbs, B.W. Williams, Fluorescence in membranes, in: J.R. Lakowicz (Ed.), Topics in Fluorescence Spectroscopy, vol. 3, Plenum Press, New York, 1992, pp. 231-271.

[14] (a) C.R. Mateo, M.P. Lillo, J. González-Rodríguez, A.U. Acuña, Molecular order and fluidity of the plasma membrane of human platelets from time-resolved fluorescence depolarization, Eur. Biophys. J. 20 (1991) 41-52;

(b) C.R. Mateo, M.P. Lillo, J. González-Rodríguez, A.U. Acuña, Lateral heterogeneity in human platelet plasma membrane and lipids from time-resolved fluorescence of t-parinaric acid, Eur. Biophys. J. 20 (1991) 53-59.

[15] K. Kinoshita, S. Kawato, A. Ikegami, A theory of fluorescence polarization decay in membranes, Biophys. J. 20 (1977) 289305.

[16] G. Lipari, A. Szabo, Effect of vibrational motion on fluorescence depolarization and nuclear magnetic resonance relaxation in macromolecules and membranes, Biophys. J. 30 (1980) 489-506.

[17] W. van der Meer, H. Pottel, W. Herreman, M. Ameloot, H. Hendrickx, H. Schröder, Effect of orientational order on the decay of the fluorescence anisotropy in membrane suspensions, Biophys. J. 46 (1984) 515-523.

[18] F. Jähnig, Structural order of lipids and proteins in membranes: evaluation of fluorescence anisotropy data, Proc. Natl. Acad. Sci. U. S. A. 76 (1979) 6361-6365.

[19] K.R. Naqvi, A.U. Acuña, Polarized radiation and dynamics of biological systems, in: S. Fraga (Ed.), Studies in Physical and Theoretical Chemistry, vol. 77, Elsevier, Amsterdam, 1992, pp. 246-264.

[20] D. Toptygin, L.J. Brand, Determination of DPH order parameter in unoriented vesicles, J. Fluoresc. 5 (1995) 39-50.

[21] B. Lentz, Membrane 'fluidity' from fluorescence anisotropy measurements, in: L. Loew (Ed.), Spectroscopic Membrane Probes, vol. 1, CRC Press, Boca Raton, Florida, 1988, pp. 13-41.

[22] B. Hudson, S.A. Cavalier, Studies of membrane dynamics and lipid-protein interactions with parinaric acid, in: L. Loew (Ed.), Spectroscopic Membrane Probes, vol. 1, CRC Press, Boca Raton, Florida, 1988, pp. 43-62.

[23] C.R. Mateo, A.A. Souto, F. Amat-Guerri, A.U. Acuña, New fluorescent octadecapentaenoic acids as probes of lipid membranes and protein-lipid interactions, Biophys. J. 71 (1996) 2177-2191. 
[24] L. Kuerschner, C.S. Ejsing, K. Ekroos, A. Shevchenko, K.I. Anderson, C. Thiele, Polyene-lipids: a new tool to image lipids, Nat. Meth. 2 (2005) 39-45.

[25] (a) E. Quesada, A.U. Acuña, F. Amat-Guerri, New transmembrane polyene bolaamphiphiles as fluorescent probes in lipid bilayers, Angew. Chem., Intl. Ed. 40 (2001) 2095-2097;

(b) E. Quesada, A.U. Acuña, F. Amat-Guerri, Synthesis of carboxyltethered symmetric conjugated polyenes as fluorescent transmembrane probes of lipid bilayers, Eur. J. Org. Chem. (2003) 1308-1318.

[26] A.A. Souto, A.U. Acuña, F. Amat-Guerri, A general and practical synthesis of linear conjugated pentaenoic acids, Tetrahedron Lett. 32

(1994) 5907-5010.

[27] W.E. Blumberg, R.E. Dale, J. Eisinger, D.M. Zuckerman, Energy transfer in tRNAPhe(yeast). The solution structure of transfer RNA, Biopolymers 13 (1974) 1607-1620.

[28] J.M. Beechem, E. Gratton, M. Ameloot, J.R. Knutson, L. Brand, The global analysis of fluorescence intensity and anisotropy decay data: second-generation theory and programs, in: J.R. Lakowicz (Ed.), Topics in Fluorescence Spectroscopy, vol. 2, Plenum Press, New York, 1991, pp. 241-305.

[29] B.S. Hudson, B.E. Kohler, K. Schulten, Linear polyene electronic structure and potential surfaces, in: E.C. Lim (Ed.), Excited States, vol. 6, Academic Press, New York, 1982, pp. 1-95.

[30] W.G. Boowman, A.C. Jones, D. Phillips, P. Thibodeaou, C. Friel, R.L. Christensen, Fluorescence of gaseous tetraenes and pentaenes, J. Phys. Chem. 94 (1990) 7429-7434.

[31] B.E. Kohler, V. Terpougov, Electronic states of linear polyenes: high resolution spectroscopy of cis- and trans-1,3,5,7,9decapentaene, J. Phys.Chem. 108 (1998) 9586-9593.

[32] E. Quesada, Ph.D. Thesis, Universidad Autónoma (Madrid, 2000).

[33] J.F. Nagle, S. Tristram-Nagle, Structure of lipid bilayers, Biochim. Biophys. Acta, Rev. Biomembr. 1496 (2000) 159-195.

[34] L. Bagatolli, E. Gratton, T. Khan, P.L.G. Chong, Two-photon fluorescence microscopy studies of bipolar tetraether giant liposomes from thermophilic archaebacteria Sulfolobus acidocaldarius, Biophys. J. 79 (2000) 416-425.

34 A.U. Acuña et al. / Biophysical Chemistry 122 (2006) 27-35 ARTICLE IN PRESS

[35] (a) Q.-Y. Shang, X. Don, B.S. Hudson, Off-axis orientation of the electronic transition moment for a linear polyene, Nature 352 (1991) 703-705; (b) R.R. Birge, M.Z. Zgierski, L. Serrano-Andrés, B.S. Hudson, Transition dipole orientation of linear polyenes: semiempirical models and extrapolation to the infinite chain, J. Phys. Chem., A 103 (1999) 2251-2255.

[36] D.A. Van der Sijs, E.E. Van Fassen, Y.K. Levine, The interpretation of the fluorescence anisotropy decay of probe molecules in membrane system, Chem. Phys. Lett. 216 (1993) 559-565.

[37] A.J.C. Fulford, W.E. Peel, Lateral pressures in biomembranes estimated from the dynamics of fluorescent probes, Biochim. Biophys. Acta, Biomembr. 598 (1980) 237-246.

[38] L.W. Engel, F.G. Prendergast, Values for and significance of order parameters and 'cone angles' of fluorophore rotation in lipid bilayers, Biochemistry 20 (1981) 7338-7345.

[39] K. Kinoshita, A. Ikegami, S. Kawato, On the wobbling-in-cone analysis of fluorescence anisotropy decay, Biophys. J. 37 (1982) 461-464.

[40] G.S. Harms, M. Sonnleitner, G.J. Schütz, H.J. Gruber, Th. Schmidt, Single-molecule anisotropy imaging, Biophys. J. 77 (1999) 2864-2870.

[41] M.X. Fernandes, M.L. Huertas, M.A.R.B. Castanho, J. García de la Torre, Simulation of the distribution and diffusion of a rigid amphipatic particle embedded in a model membrane, Biophys. Chemist. 79 (1999) 41-53.

[42] P.B. Moore, C.F. Lopez, M.L. Klein, Dynamic properties of a hydrated lipid bilayer from multinanosecond Molecular Dynamics Simulation, Biophys. J. 81 (2001) 2484-2494. 